\title{
Tracheal Schwannoma Presenting as Status Asthmaticus in a Sixteen-Year-Old Boy: Airway Considerations and Removal With the $\mathrm{CO}_{2}$ Laser
}

\author{
Daniel J. Weiner, MD, ${ }^{1}$ Robert A. Weatherly, MD, $^{2}$ Michael A. DiPietro, ${ }^{2},{ }^{3}$ and \\ Georgiana M. Sanders, MD ${ }^{1 *}$
}

\begin{abstract}
Summary. A 16-year-old with clinical features of atypical asthma is presented, with a description of the workup leading to the diagnosis of an intratracheal mass. The mass was visualized with a flexible fiberoptic bronchoscope, then surgically removed through a rigid bronchoscope using a $\mathrm{CO}_{2}$ laser. We believe this is the first report of resection using this technique. A discussion of tracheal neurilemmomas (schwannoma) is included. This case reinforces the age-old adage that "not all that wheezes is asthma." Pediatr Pulmonol. 1998; 25:393-397.

() 1998 Wiley-Liss, Inc.
\end{abstract}

Key words: asthma; intratracheal tumor; schwannoma; $\mathrm{CO}_{2}$ laser; neurilemmoma.

\section{CASE HISTORY}

A 16-year-old white male was admitted to our institution with complaints of cough, wheezing, and decreasing exercise tolerance since treatment for a pneumonia 11 months earlier. One month prior to admission, spirometry at an outside institution was interpreted as consistent with severe obstructive airway disease. He was begun on an albuterol metered dose inhaler and a 10-day course of oral erythromycin. Despite this, he developed increasing chest tightness and dyspnea. At an outside emergency room he was given three nebulized mist treatments and intravenous methylprednisolone with minimal relief and then begun on a 3-day course of oral methylprednisolone. Because of increasing cough productive of white sputum, he was seen in the outside ER twice the following day and again treated with nebulized albuterol and intravenous methylprednisolone. He was brought to our institution two days later because of persisting dyspnea, cough, and development of low-grade fever. He admitted to 3-4 pillow orthopnea with aggravation of coughing while supine.

Medications on admission were the albuterol inhaler and oral methylprednisolone (day 3). He had smoked onehalf pack of cigarettes per day for the last 3 years, but quit 6 months prior to presentation due to difficulty in breathing.

Initial physical examination revealed a temperature of $37.3^{\circ} \mathrm{C}$, HR of $102 / \mathrm{min}, \mathrm{RR}$ of $28-32 / \mathrm{min}$, BP of $100 / 70$ $\mathrm{mmHg}$, and an oxygen saturation of $95 \%$ on room air. He was a very thin young man, sitting up and leaning forward in moderate respiratory distress. HEENT examination revealed shotty anterior cervical lymphadenopathy bilaterally and crepitus in the right neck. Lung auscultation indicated very poor air movement, a prolonged (c) 1998 Wiley-Liss, Inc. expiratory phase, and diffuse inspiratory and expiratory wheezing. Heart, abdomen, skin, and neurological evaluation was within normal limits. The initial radiograph showed massive symmetrical hyperinflation, minimal peribronchial thickening, but no infiltrates (Fig. 1). Laboratory studies showed an $\mathrm{ABG} \mathrm{pH} 7.40 / \mathrm{pCO}_{2} 37$ torr/p02 72 torr $/ \mathrm{HCO}_{3}^{-} 23 \mathrm{mEq} / \mathrm{L} /$ oxygen saturation of $94 \%$, a WBC of $11.2 \times 10^{3} / \mathrm{mm}^{3}$ (61\% segmented polymorphs, $1 \%$ bands, $29 \%$ lymphocytes), hemoglobin concentration $15.1 \mathrm{~g} / \mathrm{dL}$, and a platelet count of $325,000 / \mathrm{mm}^{3}$. Electrolytes and sweat chloride levels were normal. The patient was admitted and begun on frequent nebulized treatments with albuterol and intravenous methylprednisolone. Diagnostic considerations at this time included bronchiolitis obliterans, asthma, hypersensitivity pneumonitis, and alpha-1 antitrypsin deficiency.

\footnotetext{
${ }^{1}$ Department of Pediatrics, University of Michigan Medical Center, Ann Arbor, Michigan.

${ }^{2}$ Department of Otolaryngology, University of Michigan Medical Center, Ann Arbor, Michigan.
}

${ }^{3}$ Department of Radiology, University of Michigan Medical Center, Ann Arbor, Michigan.

Current address for Dr. Daniel J. Weiner: Pediatric Pulmonology, St. Christopher's Hospital for Children, Philadelphia, PA.

*Correspondence to: Dr. Georgiana M. Sanders, Department of Pediatrics, Division Pediatric Pulmonology, D1213 MPB, Box 0718, 1500 E. Medical Center Drive, Ann Arbor, MI 48109-0718.

Received 27 March 1995; accepted 24 December 1997. 


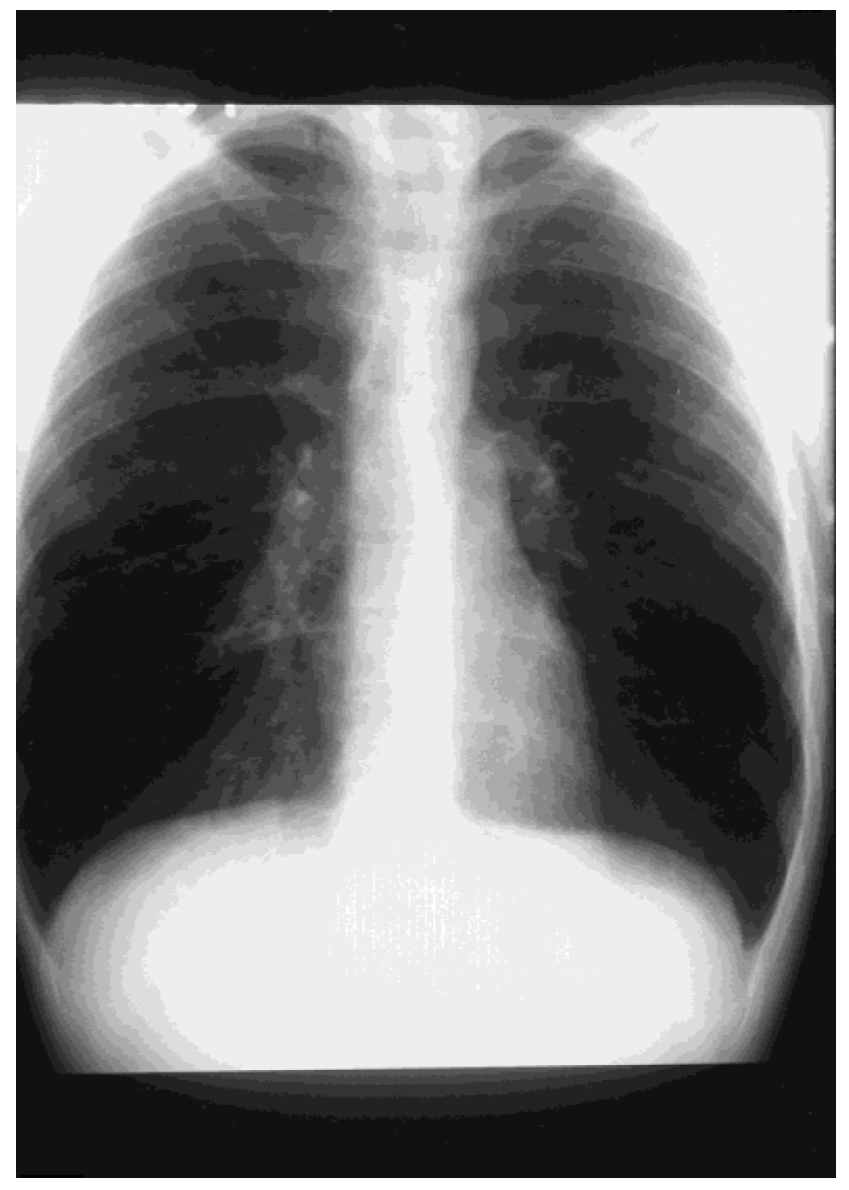

Fig. 1. Chest X-Ray (PA view) at admission showing marked, bilateral hyperinflation.

Spirometry (Fig. 2a) revealed severe limitation of both inspiratory and expiratory flows (as demonstrated by flattening of both loops), consistent with a fixed airway obstruction. Chest CT revealed a lesion apparently within the lumen of the trachea (Fig. 3) which could, in retrospect, be seen as a round lesion on the plain film and was better visualized with magnified airway views and airway fluoroscopy (Fig. 4).

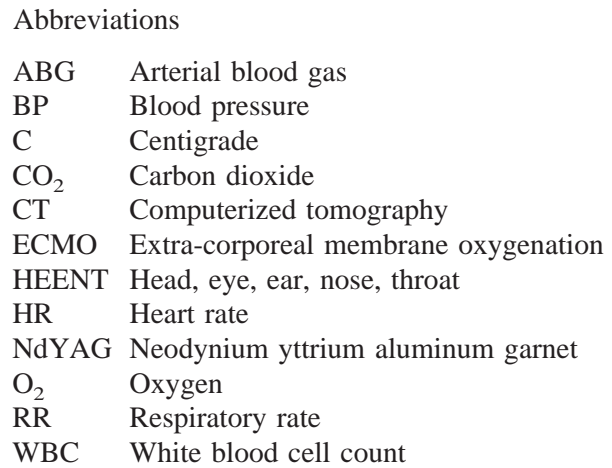

\section{TREATMENT}

On the CT evaluation, the mass appeared to be entirely intraluminal, which lessened the possibility that large feeding vessels were supplying the tumor directly and suggested that the trachea would be somewhat mobile and distensible, although it was not possible to determine the exact location of the patient's remaining airspace around or through the lesion. It was determined that direct inspection of this area would be necessary to view the patient's airway and secure it distally using a long cuffed endotracheal tube. The decision was made to proceed with bronchoscopy for airway control and either debridement or removal of the mass, as dictated by the circumstances at the time of inspection. Concerns regarding the anesthetic technique and the options available in relation to the procedure itself were discussed at length. ${ }^{3,4,5} \mathrm{We}$ chose to induce anesthesia with a small amount of intravenous sedation (midazolam, droperidol, fentanyl, and atropine), combined with a local anesthetic (lidocaine and cocaine) to the larynx and pharynx proper. The patient was allowed to ventilate spontaneously during this and all subsequent portions of the procedure. Fiberoptic bronchoscopic intubation was performed, initially, with the patient in a sitting position to maintain patency of the airway and allow for visualization of the tumor and directed intubation to secure the airway. Once the airway was secure, the patient was placed in the supine position and the rigid bronchoscope was inserted to allow for tumor removal with the $\mathrm{CO}_{2}$ laser.

With the patient sitting and breathing spontaneously, a 2.7-mm diameter fiberoptic bronchoscope was threaded through a 4.5-mm cuffed reinforced endotracheal tube introduced orally. The visualized larynx was normal. The trachea was markedly obstructed by a $1.5 \times 2.0 \mathrm{~cm}$ smooth, tan-colored, well-circumscribed mass. The surface of the mass was covered with several small, discrete vessels but the tumor was not pulsatile nor friable and did not otherwise demonstrate any signs of excessive vascularity, confirming our initial impression. A small, slit-like airway was seen posterolaterally along the right side of the trachea (Fig. 5). The bronchoscope was advanced distally around the tumor. The carina and both mainstem bronchi were unobstructed, although they contained a moderate amount of mucoid secretions. The endotracheal tube was advanced over the bronchoscope into the distal trachea and the endotracheal tube cuff was inflated just beyond the tracheal mass, securing the airway. The fiberoptic bronchoscope was then removed and the patient was placed in the supine position.

Although the endotracheal tube allowed adequate ventilation, we were unable to visualize the tumor with a rigid bronchoscope while the endotracheal tube was in place; it was therefore removed and the rigid instrument 

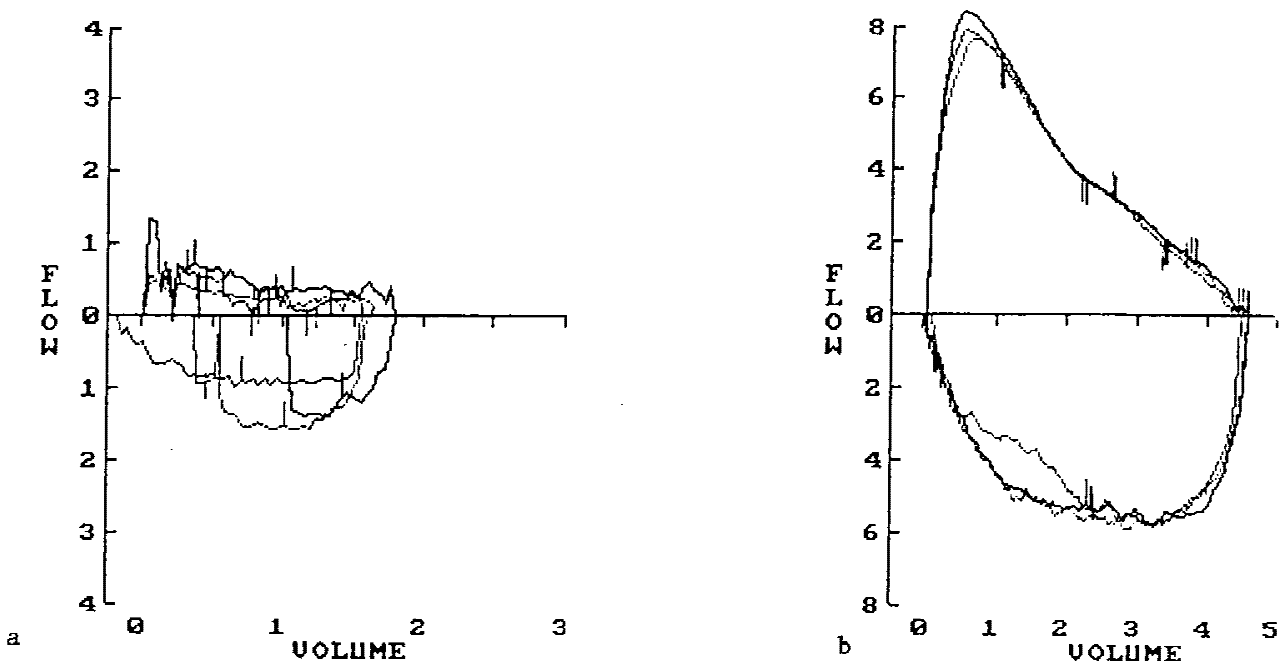

Fig. 2. Flow-volume loops obtained before (a) and 2 weeks postsurgery (b). Note the different scales on the flow axis.

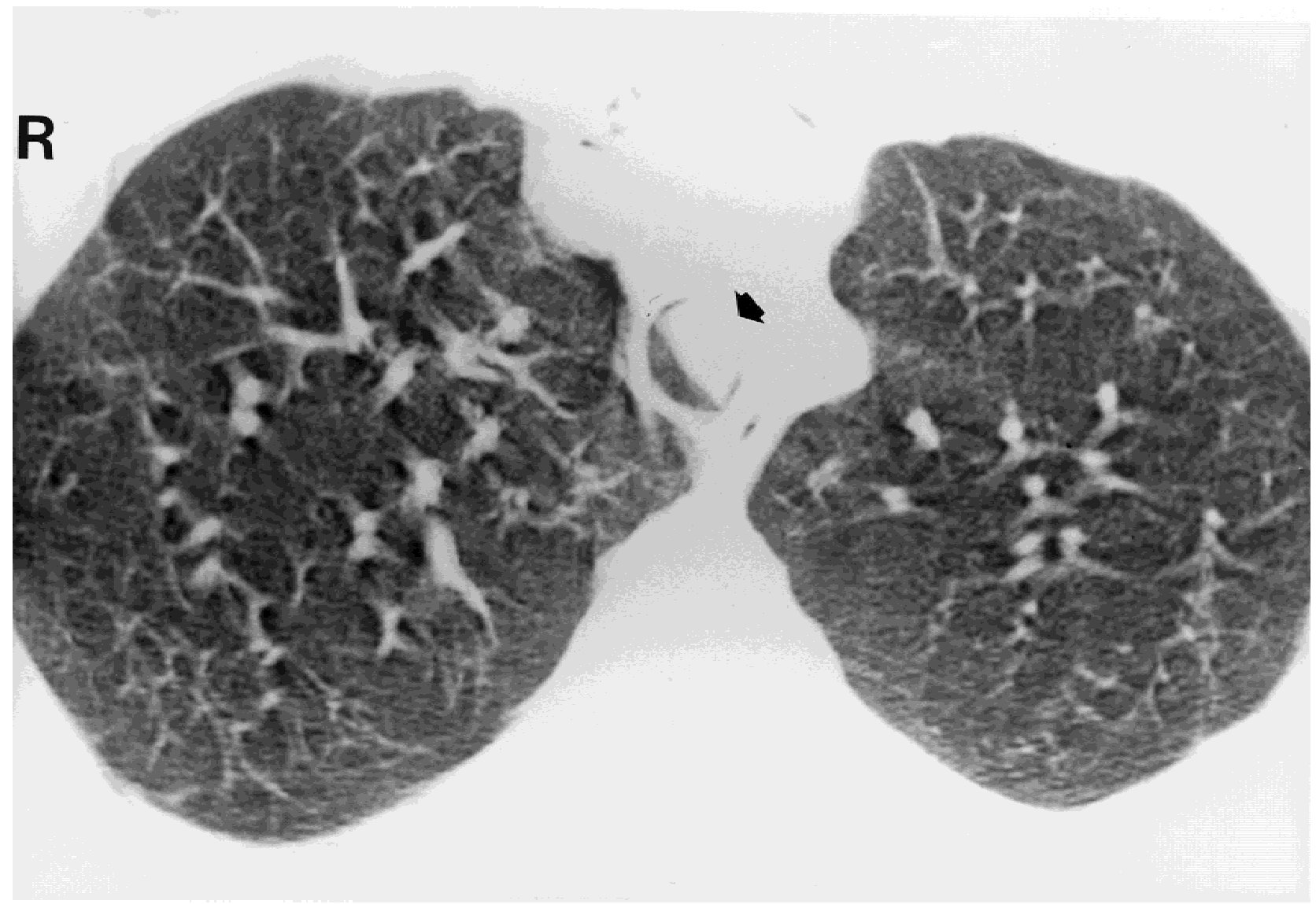

Fig. 3. Chest CT shows a soft tissue mass within the lumen of the distal trachea (arrow).

was positioned above the tumor for airway maintenance, tumor removal, and direct tamponade, if necessary in the event of massive bleeding. ${ }^{6}$ It was noted that a satisfactory airway remained after the removal of the endotracheal tube and it did not become necessary to pass the rigid bronchoscope beyond the tumor mass to ensure ventilation. The rigid bronchoscope/fiberoptic telescope system was directly connected to a $\mathrm{CO}_{2}$ laser with a micromanipulator to provide a means by which tumor tissue could be cut and cauterized for more precise debulking.

The $\mathrm{CO}_{2}$ laser beam was directed down the lumen of 


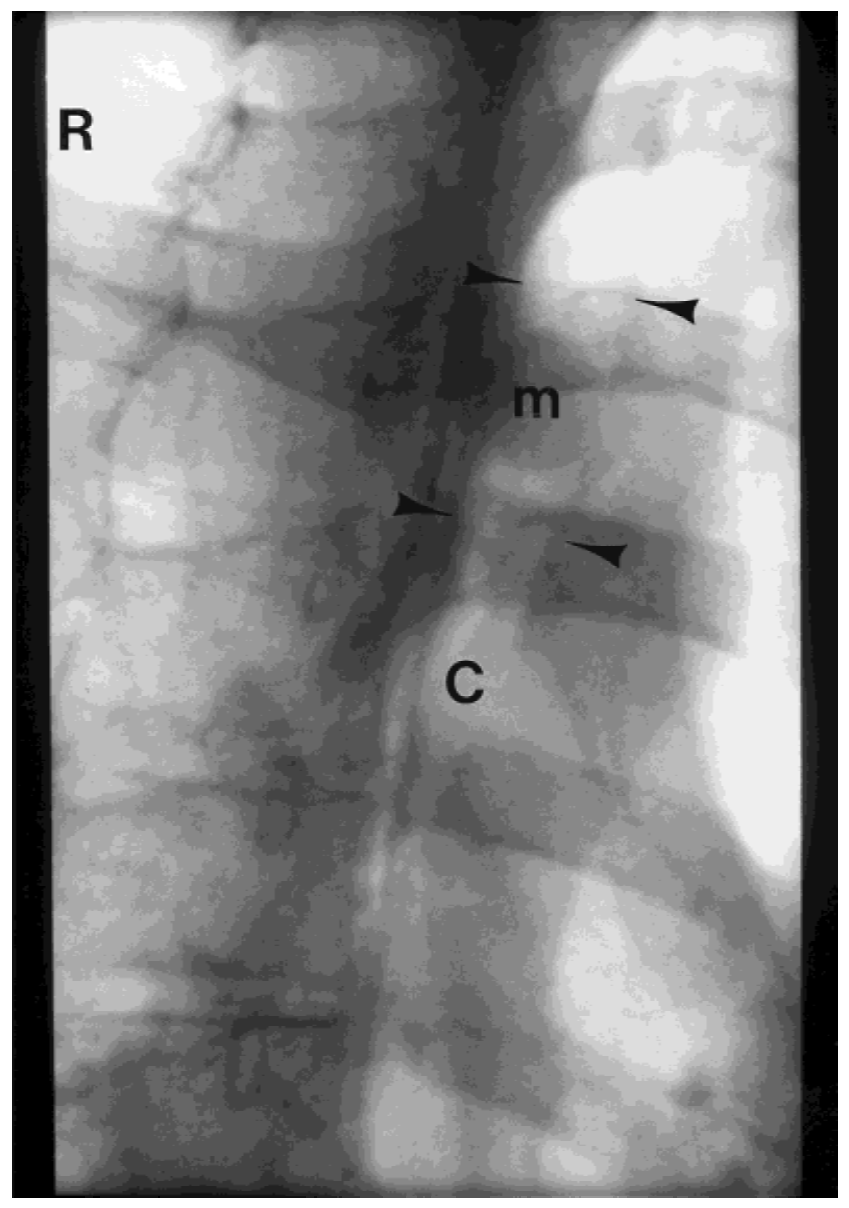

Fig. 4. Airway fluoroscopy reveals a mass $(m)$ sitting in the distal airway (outlined by arrows) above the carina (c).

the bronchoscope using a superpulse mode to provide deep tissue cutting power with some surface cauterization, while creating relatively little dissipated thermal energy. Tumor removal proceeded smoothly in a cookiebite fashion after several test pulses were applied to ensure that the mass was not hypervascular. The patient's ongoing spontaneous respiratory effort eliminated the need to advance the bronchoscope past the tumor to provide for enhanced oxygen delivery, thus expediting tissue removal. Although the initial goal was improvement in the cross-sectional area of the trachea, it became evident that macroscopic tumor removal was possible, as the attachment of the mass to the tracheal wall was stalk-like along the left anterolateral region of the distal trachea.

\section{PATHOLOGY}

Light microscopy revealed a spindle cell tumor with an inflammatory cell infiltrate; the degree of mitotic activity suggested a benign lesion. This was thought initially to represent a myofibroblastic tumor. However, subsequent immunohistochemical testing demonstrated

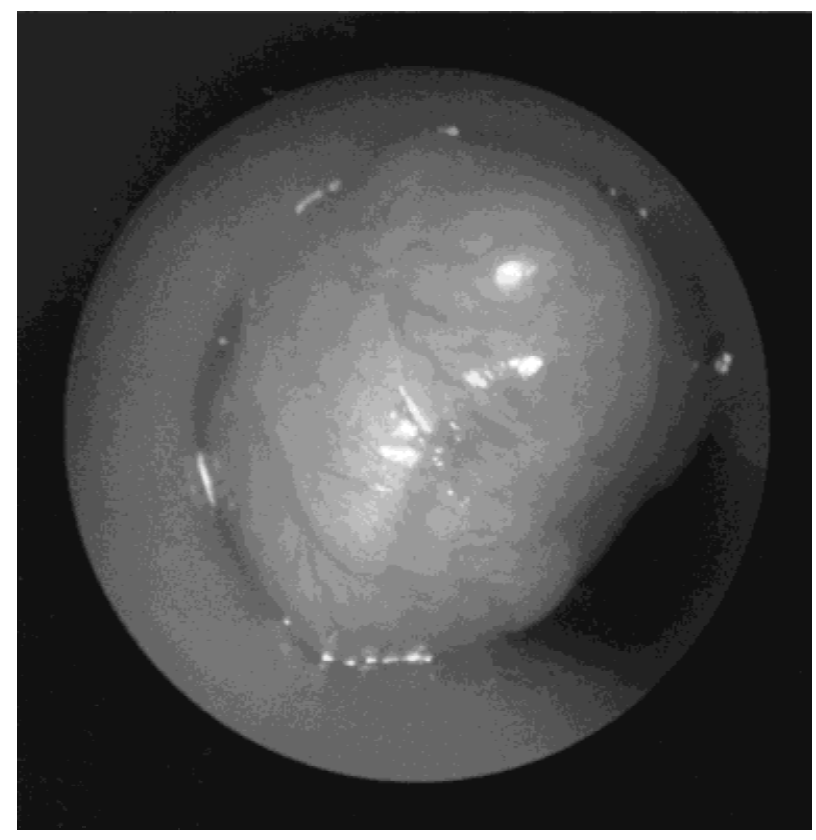

Fig. 5. Through the rigid bronchoscope, the mass is seen arising from the left side of the trachea.

that the tumor stained strongly positive with antibodies to S100 protein and vimentin, indicative of a nerve sheath tumor or schwannoma.

\section{DISCUSSION}

Tracheal tumors are uncommon in adults, and even more so in children. The differential diagnosis of a tracheal mass includes malignant (squamous cell carcinoma, adenoid cystic carcinoma, and carcinoid) as well as benign tumors, such as papilloma, hemangioma, hamartoma, and neurilemmomas and neurofibromas. ${ }^{7}$

Neurilemmomas (schwannomas) are usually single, encapsulated lesions composed of Schwann cells attached to a nerve but containing no neurons (in contrast to neurofibromas, which do contain neurons, and are usually multiple, unencapsulated lesions). Additionally, while neurofibromas are commonly associated with neurofibromatosis and occasionally undergo malignant degeneration, neurilemmomas do not. ${ }^{8}$ Histologically, spindle cells are arranged in either a highly cellular, palisading nuclei pattern (Antoni A) or less cellular, more fibrous pattern (Antoni B); most tumors have areas of each, ${ }^{14}$ as did our patient's tumor.

Tracheal neurilemmomas were first described in 1951 by Straus and Guckien, ${ }^{9}$ and 20 such cases have been reported to date, ${ }^{10}$ mostly in middle-age adults, but also in children. ${ }^{8,11}$ They are most frequently located in the distal third of the trachea and present with symptoms of cough and wheezing or stridor (especially positional). Several patients (including ours) have been mistakenly 
treated for asthma. ${ }^{8,10,12}$ Due to the slow growth and rarity of these tumors, the average delay in diagnosis is 10 to 15 months from the onset of symptoms. ${ }^{13}$ By this time, it is not uncommon for $50-75 \%$ of the tracheal lumen to be occluded by tumor. ${ }^{8}$ The diagnosis of a fixed upper airway obstruction may be suggested, as in this case, by spirometry demonstrating the classical ovalshaped flow volume loops and/or by radiologic studies, and is definitively confirmed by bronchoscopy.

Due to the high degree of airway obstruction present in this patient, extensive preoperative planning (involving the pediatric pulmonology, otolaryngology, anesthesiology, and surgical services) was necessary in the event of an airway emergency during the proposed bronchoscopic examination. In the event of sudden airway obstruction, if a rigid bronchoscope could not be used for distal control of ventilation other alternatives would be necessary to provide for airway control and/or adequate ventilation. One such alternative method of providing for gas exchange in such a patient could be the use of extracorporeal membrane oxygenation (ECMO). This method has been used to obviate the need for tracheal ventilation in a patient with respiratory failure who inhaled wood shavings, allowing for successful removal of the intratracheal material with subsequent normalization of the patient's respiratory status. ${ }^{1}$ Others have speculated on its potential usefulness in cases of respiratory arrest from upper airway obstruction due to distorted or inaccessible airway anatomy, but questions remain regarding the ability to provide this modality at a moment's notice in an emergency situation. ${ }^{2}$ We discussed with members of the Pediatric Surgery service the possible use of ECMO in our patient in the event of total airway obstruction not relieved by rigid bronchoscopy. Because it was never needed, its effectiveness in this situation is still untested. Another emergency alternative to bypass the tumor, control tumor bleeding, or both, would be open thoracotomy and distal tracheotomy. The primary limitation of this approach is the amount of surgical time necessary to gain access to the mediastinum itself.

These tumors have been treated with endoscopic resection, ${ }^{8,12,14}$ operative resection through a tracheal or laryngeal fissure, ${ }^{7}$ as well as by tracheal resection. ${ }^{8}$ There is one prior report of endoscopic NdYAG laser resection. ${ }^{10}$ We believe this represents the first case report of resection with the $\mathrm{CO}_{2}$ laser. The management of individual cases will depend on the size and location of the tumor, and by the extent of extratracheal extension. These tumors have been reported to recur; in the study by Horovitz et al., ${ }^{8}$ one of five tumors recurred more than a decade after endoscopic resection. Because of this, our patient will be followed with serial pulmonary function tests and imaging tests, as indicated.

Our patient's orthopnea and positional exacerbation of his cough were not typical of asthma, and suggested an- other process. In addition, his flow-volume loop was characteristic of fixed airway obstruction. This combination of atypical symptomatology and spirometry indicated that further evaluation was necessary, leading to the correct diagnosis. Cases such as this serve to reinforce the age-old adage that "not all that wheezes is asthma." The differential diagnosis should include (but not be limited to) a foreign body in the trachea, cystic fibrosis, immune deficiencies, tracheobronchomalacia, and gastroesophageal reflux disease with aspiration. Tracheal tumors in general, and neurilemmomas in particular, represent rare causes of airway obstruction. Labeling a wheezing patient as "asthmatic" without demonstrable reversibility of airway flow obstruction may lead to a significant delay in diagnosis and proper therapy.

\section{REFERENCES}

1. Higashi K, Takeshita J, Terasaki, H, Tanoue T, Esaki K, Sakamoto M, Tsutsumi R, Kishi H, Yano T, Ootsu T. A case of acute airway obstruction with sharp sawdust particles, successfully treated by extracorporeal lung assist [in Japanese]. Kokyu to Junkan-Respir Circ. 1989; 37:329-333.

2. Kelly MF, Berenholz L, Rizzo KA, Greco R, Wolfson P, Zwillenberg DA. Approach for oxygenation of the newborn with airway obstruction due to a cervical mass. Ann Otol Rhinol Laryngol. 1990; 99:179-182.

3. Benumof I. Anesthesia for emergency thoracic surgery. In: Benumof J, ed. Anesthesia for Thoracic Surgery. Philadelphia: WB Saunders, 1995: 612-656.

4. Eisenkrati J, Neustein S. Anesthetic management of therapeutic procedures of the lungs and airway. In Kaplan J, ed. Thoracic Anesthesia. New York: Churchill Livingstone, 1991: 419-440.

5. Watson C. Endoscopic procedures of the airways, lungs and pleura. In: Cohen E, ed. The Practice of Thoracic Anesthesia. Philadelphia: JB Lipprincott, 1995: 457-495.

6. Prakash U, Freitag L. Hemoptysis and bronchoscopy-induced hemorrhage. In: Prakash U, ed. Bronchoscopy. New York: Raven Press, 1994: 227-251.

7. Stack PS, Stecker RM. Tracheal neurilemmoma: Case report and review of the literature. Head Neck. 1990; 12:436-439.

8. Horovitz AG, Khalil KG, Verani RR, Guthrie AM, Cowan DF. Primary intratracheal neurilemmoma. J Thorac Cardiovasc Surg. 1983; 85:313-317.

9. Straus GD, Guckien JL. Schwannoma of the tracheobronchial tree. Ann Otol Rhinol Laryngol. 1951; 60:242-246.

10. Rusch VW, Schmidt RA. Tracheal schwannoma: Management by endoscopic laser resection. Thorax. 1994; 49:85-136.

11. Brunel F, Fourmaintraux A, Mariette JB, Pioche D, Campinos L, Coulin P, Mauras JR. Tracheal neurinoma simulating status asthmaticus in a child. Arch Fr Pediatr. 1993; 50:319-321.

12. Robin J, Wilson AC. Polypoid neurilemmoma of the trachea: An unusual case of major airway obstruction. Aust NZ Surg. 1988; 58:912-914.

13. Karlan MS, Livingston PA, Baker DC Jr. Diagnosis of tracheal tumors. Ann Otol Rhinol Laryngol. 1973; 82:790-799.

14. Nass RL, Cohen NL. Neurilemmoma of the trachea. Arch Otolaryngol. 1979; 105:220-221.

15. Pang LC. Primary neurilemmoma of the trachea. South Med J. $1989 ; 82: 785-787$

16. Duncavage JA, Ossoff RH. Laser application in the tracheobronchial tree. Oto Clin NA. 1990; 23:67-75. 